\title{
Introducing control in an open online course
}

Conference or Workshop Item

Accepted Version

Mitchell, R. (2016) Introducing control in an open online course. In: 2016 UKACC 11th International Conference on Control, 31 Aug - 2 Sep 2016, Belfast. (ISBN:

9781467398916) Available at http://centaur.reading.ac.uk/69085/

It is advisable to refer to the publisher's version if you intend to cite from the work. See Guidance on citing.

Published version at: https://ieeexplore.ieee.org/document/7737601

All outputs in CentAUR are protected by Intellectual Property Rights law, including copyright law. Copyright and IPR is retained by the creators or other copyright holders. Terms and conditions for use of this material are defined in the End User Agreement. 


\section{CentAUR}

Central Archive at the University of Reading

Reading's research outputs online 


\title{
Introducing Control in an Open Online Course
}

\author{
R J Mitchell \\ School of Systems Engineering \\ University of Reading \\ Reading UK \\ r.j.mitchell@reading.ac.uk
}

\begin{abstract}
Begin Robotics is a successful open online course developed at the University of Reading, run on the FutureLearn platform, for which around 25,000 participants have enrolled in its first three runs. Whilst it is aimed at introducing robotics and the associated subjects of cybernetics, artificial intelligence, control and haptics to Key Stage 3 pupils, it has been taken by other groups from around the world. This paper discusses how Control Engineering is introduced in an accessible way, and how it has been used in undergraduate degrees.
\end{abstract}

Keywords—robotics, online learning, control

\section{INTRODUCTION}

Massive Open Online Courses, or MOOCs, are the latest incarnation in 'distance education'. MOOCs are facilitated by the internet and mobile technology and as Casey[1] notes they are a direct descendant of correspondence courses which were facilitated by postal services. The first MOOC "Connectivism and Connective Knowledge" was offered by the University of Manitoba in 2008[2]. Since then many institutions throughout the world, not just universities, have offered courses over a wide range of topics, running on platforms including Coursera (Stanford University), edX (Harvard University and MIT) and more recently FutureLearn (Open University).

One of the uses of MOOCs for universities is as a marketing tool, highlighting to potential students the expertise of the university in a particular field. The first MOOC at Reading, Begin Programming, teaches students to write a game on a phone, and $10 \%$ of students on our undergraduate courses were influenced to come to Reading by the MOOC.

Robotics has been used successfully as a recruitment tool for many years[3]. This included robots in interactive talks at Schools, competitions (often funded by Public Understanding of Science projects), a collectable fortnightly magazine, exhibitions in museums, and appearances in the media. Key to the success of these activities is presenting the material in an engaging accessible manner, and allowing students to interact with the robots. Talks to schools were made effective by explaining the context in which robots are studied.

As such, it was decided that a MOOC on robotics could be an excellent opportunity to highlight Reading, its robotics work and the different degrees then on offer. As the first MOOC was Begin Programming, the new one was called Begin Robotics, and it has run in June 2015, September 2015 and February 2016 during the first year lectures on robotics.
Begin Robotics introduces robotics in the context of the associated fields of cybernetics, artificial intelligence and control engineering. It differs from other online robotics courses which are often at a higher level or focus on one robot (such as Lego Mindstorms).

This paper concentrates specifically on the control engineering aspects, how they are made accessible to the target audience, and considers also how aspects are included in the undergraduate courses. In the next section, some relevant pedagogy is introduced. Next an overview of Begin Robotics is presented, followed by sections describing the interactive web pages used for robot exercises and for demonstrating control engineering concepts. Some user comments are then provided.

\section{Pedagogy OF MOOCs}

In designing the MOOC, we adhered to the first principles of instruction, noted by Margaryan et al [4] and proposed by Merrill[5]. For instance, as regards being "problem centred" the subject matter of the MOOC is naturally set in the context of real-world problems, and this is reinforced by the inclusion of actual robots performing various tasks. Regarding "Activation", tasks were planned to help learners incorporate new knowledge into existing skills. For "Application", feedback control was introduced in the context of control of a boat and then applied to speed control of a robot, and even temperature control. Exercises where incorporated in each week where students commanded robots to do various tasks.

Miller at al [6] highlight best practices in online teaching which we adopted. The instructor has a strong presence during the course, monitoring and responding to comments (a specific example is detailed later). A learning community is created with collaborative experiences. So, for instance, topics are presented and then the participants are invited to comment, adding their own experiences, responding to others, which contribute to the collective knowledge.

\section{BEGIN ROBOTICS}

Begin Robotics is a four week course. The course, robotics and the tools used are introduced in week 1: feedback is shown to be crucial. The second week considers the anatomy of a robot, its sensors, actuators, 'brain' and power supply. The third week covers feedback for control and for interaction between robots and between humans and robots. The final week includes feedback for learning in robotic systems. 


\section{A. Introduction to Robotics}

Figure 1 depicts the image the author has developed for introducing the concepts of the course in a coherent way. It is modelled on MC Escher's wood carving of 'Other Worlds', which depicts one moonscape from different perspectives, whereas here different aspects of robotics are shown. The author has used a similar image in talks to schools, showing cybernetics in a 'different perspective'.

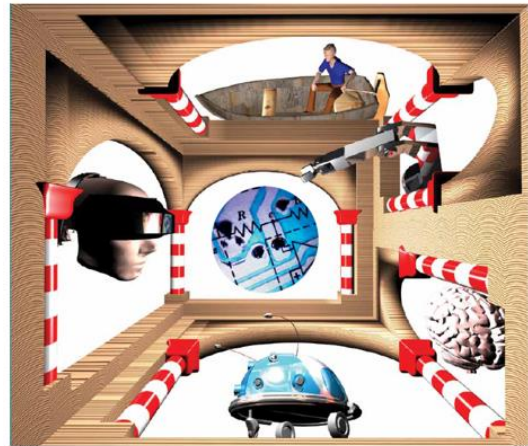

Figure 1 Escher inspired image for introducing robotics

The top arch depicts a steersman which gave the name to the subject of cybernetics and illustrates feedback control. In an introductory video the concept of feedback control is introduced in the context of a steersman, and then shown to apply to steering of a car, driving a car at the right speed, ensuring a mobile robot's speed is constant as it travels over different surfaces, etc.

To the right is an arch depicting a manipulator robot. At the bottom is an arch with a mobile robot, in fact the Cybot which featured in the Real Robotics magazine series [7].

It is then noted that a steersman is not born knowing how to steer a boat, rather he has to learn, Learning is then considered as a feedback process. The brain in the arch on the right is used to indicate learning.

On the left side is an image of someone wearing a virtual reality headset. This, it is explained, is an example of humancomputer interaction, which is another feedback process. It is noted that interaction also occurs between machines, such as mobile robots swarming together.

The image in the centre depicts electronics. These, it is explained, are crucial in the operation of robots, facilitating sensor and actuator circuits - it is noted that such circuits are often also feedback systems.

\section{B. Robot for the course}

Many robots feature in Begin Robotics. There is a long history of mobile robots at Reading, including the 'seven dwarves' [3], the 'cybot', the 'rover' used in the Part 2 cyber challenge [8], as well as various robots which had been produced as undergraduate projects. A Baxter robot is also at Reading, a modern industrial robot which has been designed to allow safe interaction between humans and robots. A popular feature of each week of the course is a 'meet the robots' section where one or more of these robots is shown.
Initially it was hoped that a mobile robot would be available which participants could buy and so experiment on. None of our existing robots were quite right, and so a new robot was designed, the aim of which was to provide as many features as possible, while being sufficiently cheap. Not every participant would want such a robot, so the course was designed so that such a robot was not a necessity. In the end, although several robots were built, logistical considerations meant that they were not available for purchase - yet!

The robot designed for the course is called ERIC, being an acronym for electronics, robotics, intelligence and cybernetics. This is a two wheeled robot, between which are mounted the batteries, motors and the circuit board. The board has on it a wide range of sensors including infrared for object detection, a $3 \mathrm{D}$ accelerometer, a microphone, a loudspeaker, motor drivers and speed sensing circuitry. Figure 2 shows a computer representation of ERIC, with the wheels, circuit board, motors and battery pack depicted.

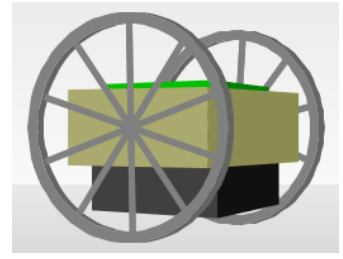

Figure 2 ERIC Robot used in the course

ERIC provides various opportunities for feedback control. The obvious one is speed control for each wheel. An interesting one is associated with the unit comprising the circuit board, motors and battery pack. As ERIC has only two wheels and no caster wheel, when it accelerates the unit rotates about the axis between the two wheels - it is an interesting control problem to dampen this oscillatory motion. ERIC features in exercises each week, although robots such as the Rover and Baxter are also demonstrated as appropriate.

\section{Interactive Web Pages}

An important element in a course such as this, as well as in other courses, is the opportunity to try out some of the ideas presented. In our outreach activities with robots, as well as a Part 1 laboratory experiment, this has been achieved by a simulator of a mobile robot moving around an environment where the student has to program its actions under different conditions. Then, when the robot performs suitably in the simulation, the code is downloaded onto the actual robot.

For Begin Robotics, users should be able to command robots and a suitable mechanism is needed. For Begin Programming, a major hurdle is the requirement in the first week to download a development system - this should be avoided. FutureLearn courses can be taken on desktops, laptops or mobile devices such as tablets or even phones. As such, it was decided that the simplest mechanism for users was to provide a series of interactive web pages which the users can link to during the course. These were written in Javascript, so that all users need is a sufficiently modern browser (such as Internet Explorer 8 onwards, Firefox, Chrome and Safari), with Javascript enabled. 
These web pages are used in two ways. Firstly, users interact with them in the weekly exercises where they command the robot to move to achieve specific tasks. These tasks are described later. Secondly, they are used to illustrate particular aspects of the course, such as how range finding sensors work, how pulse width modulation is used or even how to dampen the oscillations of ERIC's main body. More is said of these later.

The web pages are very popular (as is described later in the section detailing feedback from the course). Some participants have been school teachers who have asked to use them in schools outside the FutureLearn environment. As such they are published separately on the University web page[9].

\section{Web Pages to Command The Robot}

The simulation environment used in Outreach activities and the undergraduate laboratory requires the student to define how the robot should move at any time on the basis of information from its sensors. The student has to complete a function in the $\mathrm{C}$ language which returns the speeds of the left and right motors based on the distance of the nearest object and whether it is closest to the left or right sensors[3]. The simulator has a built in interpreter which analyses the $C$ code generating suitable data which is stored in a look up table. This is consistent with the first year programming module where $\mathrm{C}$ and later $\mathrm{C}++$ are taught.

For the Begin Robotics MOOC, aimed at key stage 3 students, but also available to all ages with many different interests and experiences, as the focus was on robotics and not programming, this was not an appropriate way of commanding the robot (although some participants indicated that they would have liked to program the robots).

Instead, it was decided that the user would be asked to enter the speeds of the left and right motors under different conditions. This was achieved in eight different web pages which were each associated with a task in the course: there are two such tasks in each week. Each of the web pages have the interface to the user, a model of the environment in which the robot moves, the boxes into which the speeds are entered, together with suitable options and text explaining the task briefly. Associated with the pages are other javascript library files which simulate the environment and robot, process commands, etc.

Each task is preceded by a screencast which explains the task. This was generated using Camtasia and is a mixture of a Powerpoint presentation and the author demonstrating the web page in action.

The first task is aimed at familiarization. The speeds that the user enters are numbers in the range -40 to +40 . If positive then, as viewed from outside the robot, a positive speed will make the wheel turn in a clockwise direction, and a negative speed in the anticlockwise direction. As this means that, for the robot to move forwards, one speed has to be positive and one negative, the pages have a 'Reverse Left Motor' option which is equivalent to connecting the wires to the motor in reverse, which many found helpful. Figure 3 shows relevant parts of the web page for the familiarization task.

\section{EXERCISE TASKS}

See what happens when you try different values for the left and then right motor.

Work out how to make the robot go forwards/backwards and spin left/right.

Investigate the effect of ticking the "Reverse Left Motor" option.

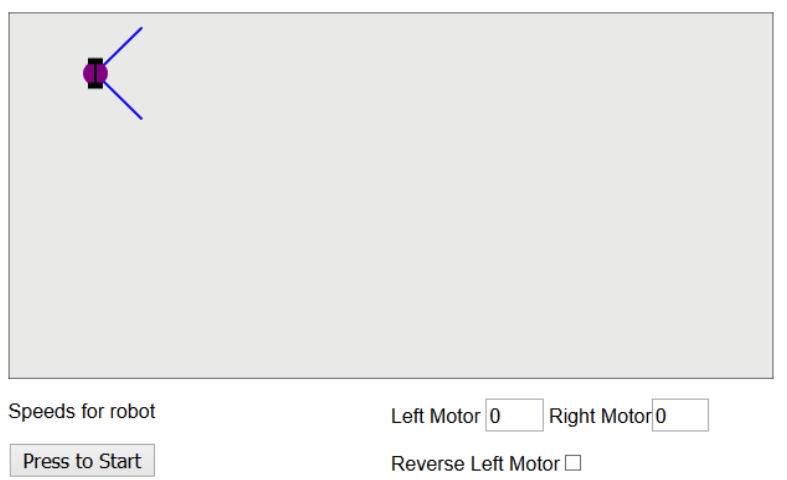

Figure 3 First Robot Exercise - initial investigation

The participant is instructed to see what happens when the speeds are 0 and 4 , then 4 and 0 and to then try different speeds and investigate the Reverse Left Motor option.

The second task in the first week is to define the speeds for specific actions: pairs of speeds are set for going forward, turning left, turning right, going backwards or stopping. Figure 4 shows the commands, which are displayed below the arena.

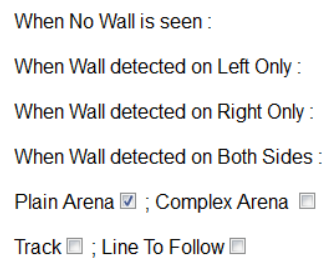

Figure 4 Second Robot Exercise - commands initially

Initially the user defines the speeds and tests them. Then the 'Track' option is selected and the arena appears as in Figure 5, with a button to start racing. The participant steers the robot as fast as possible from the red start area top left to the green end area near the middle. The time between the user starting the race and when the robot arrives is then displayed. Users are invited to post their fastest time.

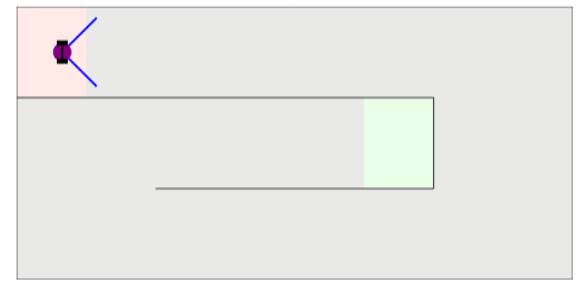

Figure 5 Second Robot Exercise race track

The first exercise of the second week is to define the motor speeds depending on the information from its two sensors. The speed pairs are defined for when no wall is seen, one is detected on the left only, one on the right only and when a wall is 
detected by both sides. These are tested initially on the same arena as shown in Figure 3, then a more complex arena as shown in Figure 5, then on the race track - where the user has to race as before (and compares the result with that in the previous week, and then (by changing the rules slightly) in the revere direction.

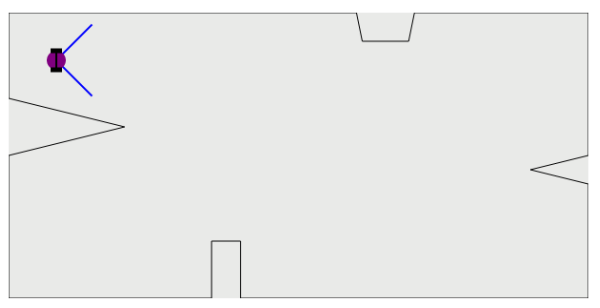

Figure 6 More complicated arena.

Finally the user sets Line To Follow mode, and the arena appears as shown in Figure 7, and then has to define the speeds for when both sensors detect the line, the left or right sensor only detects a line, or neither sensors detect the line. They find that the speeds already defined still work.

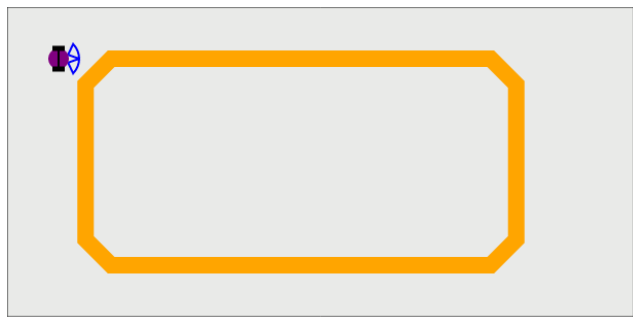

Figure 7 Arena for Line Following

For the second exercise in week 2, two lights are positioned in the arena, each sensor can detect whether a light cannot be detected, is some distance away or is close to the sensor. The tasks are for the robot to steer towards a light when it sees one, and later to veer away if it gets too close.

In the third week, the first page depicts a rectangle in the arena and the user enters commands for the robot to move forward, turn left or right, at a given speed for a certain time. After some investigation the user defines a string of such commands the aim of which is to move as close as possible around the rectangle. For instance, F 20100 R 325 L 315 means travel forward at speed 20 for 100 units, turn right at speed 3 for 25 units and turn left at speed 3 for 15 units.

Next the user selects the race track and works out the commands to move from the start to the end. Then a 'hilled' race track is selected, where there are rectangular regions which are 'uphill' where the robot slows and some which are 'downhill' where the robot speeds up. The user finds that the previous string does not work as the robot operates open loop. Then the user enables speed control and finds a command string which works for both the flat and hilled tracks, demonstrating the benefits of speed control.

In the second exercise there is a large object which follows an elliptical path, and the user has to command the robot to stay a fixed distance from the object.
The first exercise in week 4 has a robot with two sensors each reporting if no wall is detected, one is just detectable and one which is close. First, the user defines speeds so that the robot follows a wall. Then, a simple maze is selected (see Fig 8 ) and the user sets the speeds so that the maze is navigated.

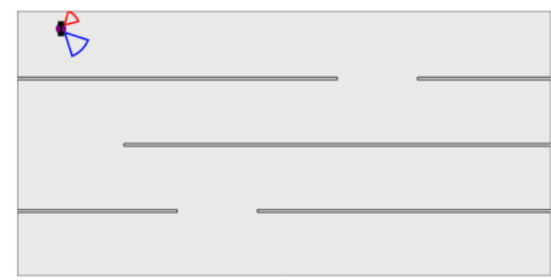

Figure 8 Arena for Maze Navigation

The second exercise simulates a Braitenberg vehicle[10], with two sensors, LS and RS, able to detect lights, with 6 neurons as shown in Figure 9, which also shows how the speeds of the two wheels are calculated.

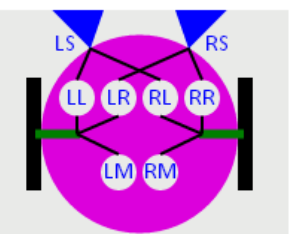

$$
\begin{aligned}
& \text { Left Speed = } \\
& \text { LM+LS*LL+RS*LL } \\
& \text { Right Speed = } \\
& \text { RM+LS*RL+RS*RR }
\end{aligned}
$$

Figure 9 Braitenberg Vechicle

The vehicle is put in an arena with two lights, and the user defines the neurons so that the robot steers towards a light or away from a light. Then the user selects line following, as per Figure 7, and defines the neurons so the robot follows the line.

\section{Web Pages to Illustrate CONCEPTS}

In addition to these interactive exercise pages, there is a series of pages which are used to demonstrate concepts. Generally these are presented as part of a screencast mixed with powerpoint slides, and then the user can try the web pages themselves.

The first such page is used to help the user as regards the wheel speeds where a positive speed means the wheel turns clockwise. In the first run of the course a $2 \mathrm{D}$ representation was used, with the right wheel in front and the left behind. The speeds were set and the user could see the directions of the wheels. For subsequent runs, a 3D model was generated, as shown in Figure 2, and the user sees how the wheels turn as different speeds are set for each motor.

In the second week the pages help explain some sensors and actuators. The first demonstrates echo location, where 'waves' are shown being emitted by the source and bouncing back from objects.

The explanation of accelerometers demonstrates how they are used on mobile phones to determine the orientation of the display. Figure 10 shows the model of a phone displayed on the web page where the user can change its angle left right and tilt ford/backwards. The lines on the screen indicate the force detected in two directions. 


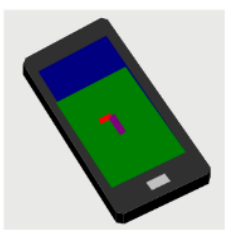

Figure 10 Image used in page illustrating accelerometers

Pulse width modulation is demonstrated as in Figure 11. The user specifies the speed at which the wheel rotates and the direction of rotation. The associated PWM signal is depicted, as is the bridge circuit which sets polarity. The PWM signal is always low at the end of the cycle, when the voltage across the additional resistor is measured which equals the back emf of the motor and hence the motor speed is determined.

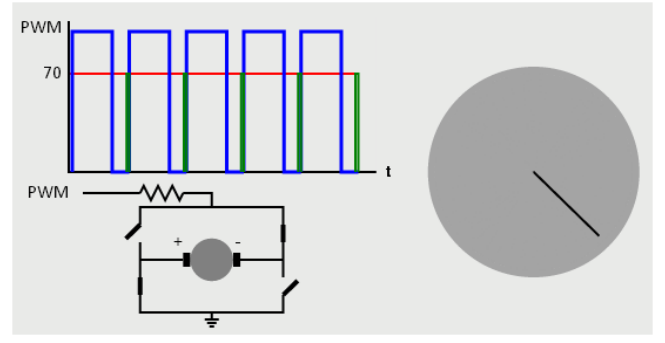

Figure 11 Image displayed to illustrate PWM

In the second run of the course one participant commented that the web pages showing the robot in $3 \mathrm{D}$, the robot moving around an arena and that illustrating PWM were useful, but a page combining them all would help further demonstrate what was happening. Figure 12 shows part of the web page the author produced the next day. As the robot moves around the arena, steering away from the walls, and accelerating in the middle, the 3D model rotates accordingly, and the PWM signals and bridge change in real time.

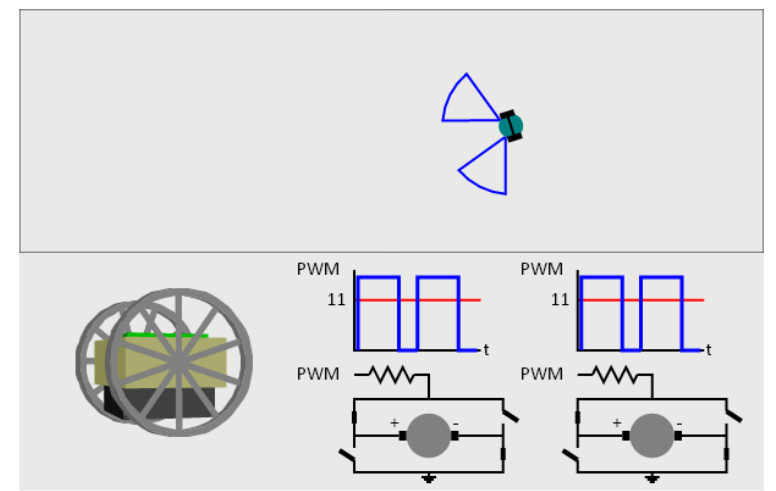

Figure 12 Image displayed to illustrate PWM and the robot

The web page to demonstrate feedback control has a robot moving over one of five 'hilly' 'landscapes', with surfaces whose friction varies. The user can run the robot over the hill open loop, and a graph showing the variation of speed over time is depicted - as shown in Figure 13. The user can then see the effect of a simple proportional control scheme with two values of controller gain, and can see that the higher gain gives a more constant speed, much nearer the desired speed.

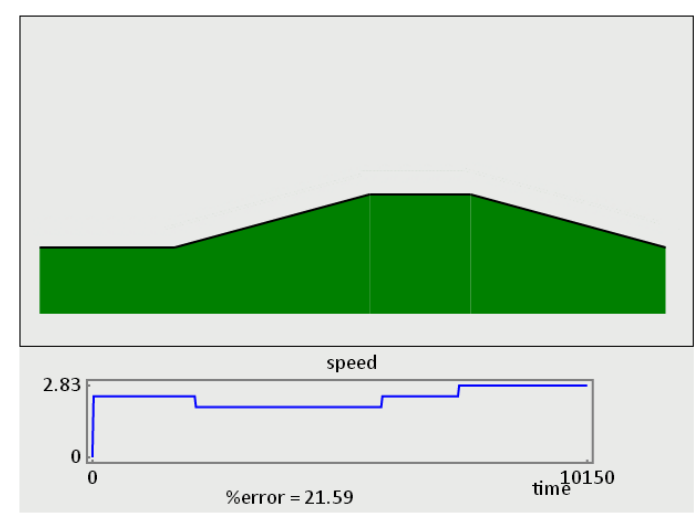

Figure 13 Page showing simple landscape and open loop speed graph

The page also allows for user control, when a speedometer is displayed below the graph and the user can speed up or slow down. Figure 14 shows the speedometer.

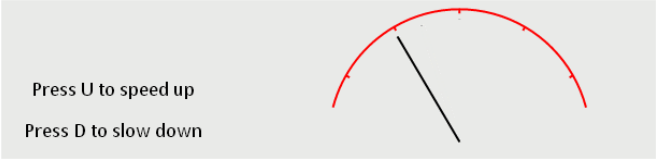

Figure 14 Simple speedometer displayed to user under the hills.

It is then noted that proportional control means that the control signal is the 'error' times a constant, whereas the user is using the error to decide whether to change speed. This it is explained is 'integral' control, and that proportional plus integral control is often used. The user can select this and see that the robot speed is then even closer to that desired.

The other main example of control illustrated is that for dampening the 'rocking' motion of the main body of the ERIC robot when it accelerates. This is due to the body rotating around the axis between the two wheels. A web page is shown illustrating the effect (as well as video images of the real robot). One key disadvantage of this motion is that the sensors used for measuring the range of other objects may point at the floor or pass over objects.

It is explained that the solution to this is to measure the angle of the body using an accelerometer and to then use the change in the angle to adjust the speed applied to the motor. The web page shows that this works.

It is important to explain how this works, and a readily understandable analogous system is used: a mass suspended under a spring. After the mass is pushed down, it will oscillate up and down, indefinitely if there is no friction, but the more friction there is, the quicker the oscillations are dampened. It is explained that friction is proportional to the speed of the mass, that is, to the change in its position, the quantity which oscillates. For ERIC, the angle of the body oscillates, and the change in this angle is fedback to dampen the motion.

The web page used is illustrated in figure 15 . For both systems, the user can specify the amount of friction/control: none, some and more. Then either system can be activated, or both together, and then the system oscillates as appropriate. In this way the concept of velocity feedback is illustrated. 


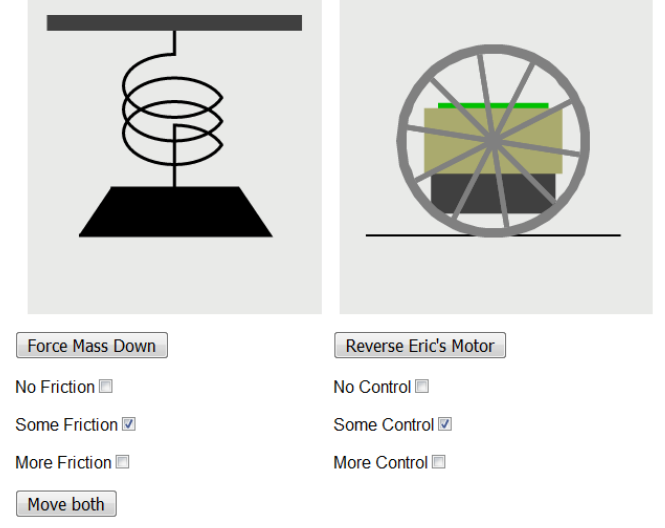

Figure 15 Analogous System illustrating velocity feedback.

\section{USE IN UNDERGRADUATE DEGREES}

The author gives an introductory course on control to first year students. This year a laboratory practical has been written around the speed control web page. Also, the dampening of the oscillatory motion of the ERIC has been used to illustrate under- and critically- damped systems. In addition, a colleague's five week course on robotics has been timed to run at the same time as the MOOC and students are encouraged to enrol on the MOOC. The impact of this is to be investigated.

\section{FEEDBACK ON THE MOOC}

Feedback on the MOOC has been very positive. Here a just a few of the comments. After the author made the simulations available, a teacher enrolled on the course said:

"Thank you Richard. I am very pleased to hear this as the simulations have been great and I think that it will be a very useful tool to allow students to experiment with the theory before using actual robots. I showed the video from week 1 where you look at the different robots you have at Reading to a group of year 9 students. They were most impressed..”.

Another participant commented: "Thank you for a truly excellent course that exceeded my expectations. By removing all the barriers to education that are still present in many online courses you allowed one to relax and concentrate on the learning. It made for a much more pleasant learning environment that encouraged exploration and experimentation. The use of simulations put this course head and shoulders above the competition."

Specifically on the control, two comments were "Was a good idea to introduce a feedback control to reduce the oscillations of ERIC, this issue had already detected previously, simulations helped me to better visualize and control reactionary already applied." And "Great explanation, I think there are many similarities between the mass-spring model and the system shown, it is a clear example of a mathematical model which can represent a system."

From FutureLearn: "The simulations Dr. Mitchell produced for the course brought the topic to life for learners and enabled them to understand \& experience robotic principles in a far more engaging way than would otherwise be possible. Encouraging learners to experiment and discuss with others is core to FutureLearn's approach to learning, and it was great to see a course adopt this so thoroughly and to such success. Most importantly, it let students have fun learning. This enjoyment was evident in hundreds of comments across the different exercises".

The University's OOC team, which supports such activity at Reading, noted that "This course went extremely well and the response from learners was overwhelmingly positive. The simulated exercises provided a useful and interesting approach and were recognised as an innovative and unique experience. The OOC team are pleased with the course statistics, particularly the high number of social learners $(42.4 \%)$ which is higher than the FutureLearn average. This reflects the course focus on learner interaction with the material". See [11] for more on social learning and such statistics.

\section{VIII.CONCLUSION}

Begin Robotics has successfully introduced robotics and control to a large audience. The interactive web pages have been particularly popular both for illustrating concepts and as practical exercises. The course has had an impact on the undergraduate degrees, and further work is planned to investigate the impact of running such a course in parallel with the first year undergraduate lectures on robotics.

\section{References}

[1] Denise M. Casey. "A journey to legitimacy: The historical development of distance education through technology." TechTrends 52, 2008, 45-51

[2] Tharindu R. Liyanagunawardena, Andrew A. Adams, and Shirley A. Williams. "MOOCs: A Systematic Study of the Published Literature 2008-2012." International Review of Research in Open and Distance Learning 14, 2013, 201-27

[3] R.J. Mitchell, K. Warwick, W. Browne, M. Gasson and J. Wyatt, 'Engaging Robots: Innovative Outreach for Attracting Cybernetics Student', IEEE Transactions on Education, Volume: 53, Issue: 1; 2010, Digital Object Identifier: 10.1109/TE.2009.2024932

[4] A. Margaryan, M. Bianco and A. Littlejohn, Instructional quality of Massive Open online Courses (MOOCs), Computers \& Education, vol 80, 2015, pp 77-83, available at: https://oerknowledgecloud.org/sites/ oerknowledgecloud.org/files/1-s2.0-S036013151400178X-main.pdf

[5] M.D. Merrill, "First principles of instruction. Educational Technology Research and Development", 50(3), 2002, 43e59.

[6] Sandra L. Miller, "Teaching an Online Pedagogy MOOC, MERLOT" Journal of Online Learning and Teaching, Vol. 11, No. 1, March 2015 .

[7] J. E. A. Wyatt, "A study of perceptions of robots through the development of a mass market consumer robotic product," Ph.D. dissertation, University of Reading, Reading, U.K., 2006..

[8] P. Minchinton, S. Gould and R. Mitchell, "The cyber challenge: a robotics project to enthuse". In: 10th IFAC Symposium Advances in Control Education, August 28 - 30 2013, University of Sheffield, Sheffield, UK, pp. 168-173.

[9] R J Mitchell https://www.reading.ac.uk/UnivRead/vr/OpenOnline Courses/Files/Simulation2/BeginRoboticsSimulationindex2.pdf 2015.

[10] V. Braitenberg. Vehicles: Experiments in synthetic psychology. Cambridge, MA: MIT Press, 1984.

[11] Simon Nelson, "Measuring our first eight courses", https://about.futurelearn.com/blog/measuring-our-first-eight-courses/ 2014. 